\title{
Editorial
}

\section{Rethinking optimality: eight concepts}

Traditional concepts of optimality in economics, operations research and management sciences are characterized by maximizing (or minimizing) a single objective function, i.e., $\operatorname{Max} f(x)$ or $\operatorname{Max} U(f(x))$ subject to constraints $X$. This usually explicates a unique solution $x^{*}$ subject to all $x \in X$, i.e., considering only the decision alternatives or options belonging to a fixed, constrained set.

As a concept, the traditional optimization model (of Max $f(x)$ subject to $x \in X$ ) is quite simple, unambiguous and 'crisp'. Although economists and engineers often use this as a well-structured reduction of the ill-structured reality, human beings, in their roles as decision makers and problem solvers, are striving to cope with reality in its fuller complexity and only rarely resort to such extreme reductionism.

People do not just minimize cost, maximize profits or maximize expected utility. Humans are always trying to balance and harmonize the competing claims of quality, quantity, time and space - all at the same time.

Human systems management, being a systemoriented approach devoted to searching for the best and the 'optimal', cannot be rooted in a simplistic single-criterion maximization with respect to constraints. Similarly, maximization, minimization and optimization processes are rarely unconstrained, unlimited or occuring out of context, striving for some absolute maxima or minima. Constraints and limitations are ever present and reflect the prevailing conditions of scarcity.

Absolute extremes, if they occur at all, are more appropriate for Utopia or certain mathematical exercises. In reality, however, (as Milton Friedman was one of the very few to argue) an economic problem exists only when scarce means are used to satisfy alternative ends. If the means are not scarce, there is no problem, but Nirvana. If the means are scarce, but there is only a single end, then the problem of how to use the means is a technological problem: no value judgments enter into its solution; only knowledge of physical and technical relationships.
So, we must learn to understand decision making as not merely a computation of the given, already constructed world (a technological problem), but as a way of the very construction of local worlds, a way of ordering of both individual and collective experience, making sense of the otherwise unordered 'chaos' of reality. Making decisions does not mean finding our ways through a fixed maze (or problemscape), but refers to the very process of constructing and reconstructing such a maze, i.e., our 'local' world of reality.

How can we do that optimally? How do we do that at all? What is optimization? Every wellstructured problem has an equally well-structured single-dimensional solution: the shortest, the safest, the cheapest or the most profitable 'path through the maze'. Finding such a path (solution) can be simple or difficult, but it is always derived or derivable from the given and fixed structure (of the maze). It is already 'there' and remains only to be uncovered or explicated (find the solution). Is this optimization?

Of course, not. Computation, calculation and orientation through a given, fixed structure is not optimization: what is given cannot be optimized; it can only be calculated.

What is the optimal path between A and B? Is it simply the best route from those already constructed (which can all be quite bad, even the best one), or does it also refer to constructing or uncovering a new route? Who is the optimizer? Is it a person who chooses the best among the given? Or is it also a person who constructs or creates the new best, de novo? If both such different and incommensurable efforts can be labeled optimization, what is then optimization? How many such 'optimizations' are there?

Here we propose that there are at least eight distinct and mutually irreducible basic concepts of optimality. They all require different modeling devices and spawn quite different problem-solving approaches. It is as if there would exist eight different operations research and management science worlds, but we have voluntarily limited ourselves to exploring and using only one of them. 
Table 1

Eight concept of optimality

\begin{tabular}{lll}
\hline \multirow{2}{*}{ Given } & \multicolumn{2}{c}{ Number of criteria } \\
\cline { 2 - 3 } $\begin{array}{l}\text { Criteria \& } \\
\text { alternatives }\end{array}$ & $\begin{array}{l}\text { Single } \\
\text { 'optimality' }\end{array}$ & Multiple \\
Criteria only & $\begin{array}{l}\text { Optimal design } \\
\text { (De novo programming) }\end{array}$ & $\begin{array}{l}\text { Optimal design } \\
\text { (De novo programming) }\end{array}$ \\
Alternatives only & $\begin{array}{l}\text { Optimal valuation } \\
\text { (limited equilibrium) }\end{array}$ & $\begin{array}{l}\text { Optimal valuation } \\
\text { (limited equilibrium) }\end{array}$ \\
'Value complex' & $\begin{array}{l}\text { Cognitive equilibrium } \\
\text { only }\end{array}$ & $\begin{array}{l}\text { Cognitive equilibrium } \\
\text { (matching) }\end{array}$ \\
\hline
\end{tabular}

What is given or determined a priori cannot be subject to optimization and thus, obviously, cannot and does not have to be optimized - it is given. What is not yet given must still be selected, chosen or identified and it is therefore, by definition, still subject to optimization. Consequently, quite different optimality concepts can be derived (see Table 1) from different distinctions between what is given and what is still to be determined.

Any criterion (measure, yardstick) or attribute is characterized by its most preferred score (or range of scores) by a given decision agent in a given context. Such contextually most preferred score is, if feasible, clearly optimal.

There is no difficulty with this concept and no need for its further elaboration. We can usually recognize the optimal amount of sugar in our coffee, optimal temperature of our body, optimal amount of water we drink daily, or optimal amount of cash we carry - as long as such preferred 'scores' are feasible.

The difficulty arises when such most preferred scores are or become infeasible, i.e., if there are explicit or implicit constraints on our criteria which prevent the achievement of the 'most preferred'.

When the optimum is infeasible, it can be approached only through maximization or minimization subject to constraints. This is virtually always the case in economics, business and management. When the constraints are fixed and there is only a single criterion, these situations become trivial.

If we relax at least some of the constraints or consider multiple criteria then the situation turns multidimensional: tradeoffs have emerged, criteria scores have to be balanced and mechanistic maximization or minimization does not suffice.

When there is only a single criterion (no matter how comprehensive, aggregate or complex) selected to describe reality, its maximization or minimization with respect to constraints is sufficient. When there are multiple criteria, much richer forms of optimality and optimization need to be explored.

\section{Eight concepts of optimality}

We have chosen to present each of the eight optimality concepts through a small example and a simple description. For a more formal treatment of modeling and computational properties consult any of the references listed.

\section{Single-objective 'optimality'}

This refers to conventional maximization (sometimes 'optimization'). Although not strictly a 'tradeoff balancing' problem, it should be included here for the sake of completeness, as a special case of bona fide optimization.

To maximize a single criterion, it is entirely sufficient to perform only technical measurement and search processes. Once $X$ and $f$ were formulated or specified, the 'optimum' (e.g., maximum) is found by computation, not via any decision or conflict dissolution processes. Search for optimality is simulated by scalarization, i.e., through assigning each alternative a number (scalar) and then identifying the highestnumbered alternative.

Example. From a list of five places $(X)$, find the one that is the cheapest $(\operatorname{Min} f)$ for vacations. 


\section{Multiobjective optimality}

Optimization is not a simple maximizing and must involve balancing and harmonizing multiple criteria. In reality, humans continually resolve conflicts among multiple criteria that are competing for their attention and assignations of importance. This corresponds to the vector optimization problem of $\operatorname{Max} f_{1}(x), \operatorname{Max} f_{2}(x), \ldots$ and $\operatorname{Max} f_{k}(x)$ simultaneously and subject to $x \in X$.

Such parallel maximization of individual functions should remain non-scalarized, separate and independent, i.e., not subject to aggregation, like forming and maximizing a superfunction $U\left(f_{1}(x), f_{2}(x), \ldots\right.$, $\left.f_{k}(x)\right)$. Such aggregate $U$ effectively reduces multiobjective optimization to a single-objective maximization. Multiple criteria, if they are to remain meaningful and functional, should be optimized (or balanced) in the vector sense, i.e., non-scalarized and in mutual competition with each other.

Example. From a list of five places $(X)$, select the one that is the cheapest $\left(\operatorname{Min} f_{1}\right)$ and safest $\left(\operatorname{Max} f_{2}\right)$ for vacations.

Remark. It is quite often argued that the difference between objectives and constraints lies only in their technical positioning: are their levels to be determined (maximized), as for the objectives, or are they given a priori, as for the constraints. In that sense costs are constraints if their a priori values are not to be exceeded and objectives if they are to be minimized.

Yet, nobody has ever argued for aggregating (or scalarizing) multiple constraints into a single, overall superconstraint. However, aggregating multiple objective functions, even if representing the same variables (like costs) is being carried out daily and goes virtually unnoticed and professionally unchallenged.

\section{Optimal system design: single criterion}

Instead of optimizing a given system $X$ with respect to selected criteria, humans often seek to form or construct an optimal system $X$ of decision alternatives, designed with respect to given criteria. Singlecriterion design is analogous to single-criterion 'optimization', but producing the best (optimal) set of alternatives $X$ at which a given, single objective function $f(x)$ is maximized subject only to the cost of design (affordability).

Example. Design an affordable list of places $(X)$ which would assure the cheapest $(\operatorname{Min} f)$ vacations.

\section{Optimal system design: multiple criteria}

This optimality form refers to the best system design with respect to blancing or harmonizing multiple criteria.

Example. Design an affordable list of places $(X)$, which assures the cheapest $\left(\operatorname{Min} f_{1}\right)$ and the safest $\left(\operatorname{Max} f_{2}\right)$ for vacations.

Instead of the set of non-dominated solutions, we now have a set (or a family) of optimal system designs, characterized by the equivalent affordability cost $\mathrm{B}$ and the differential importance of objective functions $f_{1}$ and $f_{2}$.

\section{Optimal valuation: single criterion}

All four optimization forms considered so far assume that decision criteria are given a priori. Yet, in human decision-making processes, different criteria are continually tried and applied, some are discarded, new ones added, until a proper (balanced) mix (or portfolio) of both quantitative and qualitative criteria is achieved. There is nothing more wasteful than engaging a perfectly good means $X$ towards unworthy, ineffective or only arbitrarily determined criteria (goals and objectives).

If the set of alternatives $X$ is given and fixed a priori, we speak of a problem of valuation: how should we order the alternatives? According to $f_{1}$ or $f_{2}$ or $f_{3}$ ? Which of the criteria best captures our values and purposes?

Example. Select a single criterion $(f)$, perhaps from a list $\left(f_{1}, f_{2}, f_{3}, \ldots\right)$, like entertainment, education, privacy, cost, etc., which would best evaluate a given, affordable list of places $(X)$ in order to attain most satisfactory or fulfilling (through Max or Min $f$ ) vacations.

\section{Optimal valuation: multiple criteria}

If the set of alternatives $X$ is given and fixed $a$ priori, but a set of multiple criteria is still to be selected for the evaluation and ordering of $X$, we speak of a problem of multicriteria valuation: which set of criteria best captures our complex of values and purposes? Is it $\left(f_{1}\right.$ and $\left.f_{2}\right)$ ? Or $\left(f_{3}\right.$ and $\left.f_{4}\right)$ ? Or perhaps $\left(f_{1}\right.$ and $f_{2}$ and $\left.f_{3}\right)$ ? Or even some other combination? 
Example. Select a set of criteria, perhaps a combination from a list $\left(f_{1}, f_{2}, f_{3}, f_{4}\right)$, like entertainment, education, privacy and cost, which would best evaluate a given, affordable list of places $(X)$ in order to attain most satisfactory or fulfilling (through $\operatorname{Max} f_{1}$ and $\operatorname{Max} f_{2}$ ) vacations.

\section{Optimal pattern matching: single criterion}

Here we optimize both $X$ and $f$ at the same time. There is a problem formulation representing an 'optimal pattern' of interaction between both alternatives and criteria. It is this optimal, ideal or balanced problem formulation or pattern that is to be approximated or matched by decision makers.

Example. Select a criterion $(f)$, like entertainment, education, privacy, cost, etc., and design an affordable list of places $(X)$, which would assure the most satisfactory or fulfilling (through Max or Min $f$ ) vacations.

\section{Optimal pattern matching: multiple criteria}

Pattern matching with multiple criteria is more involved and the most complex optimality concept examined so far. In all 'matching' optimality concepts there is a need to evaluate the closeness (resemblance or match) of a proposed problem formulation (singleor multi-criterion) to the optimal problem formulation (or pattern).

Example. Choose the necessary criteria $\left(f_{1}, f_{2}, \ldots\right.$, $f_{k}$ ), like entertainment, education, privacy, cost, etc., and design an affordable list of places $(X)$, which assure the most satisfying or fulfilling (for example, through $\operatorname{Max} f_{1}$ and $\operatorname{Min} f_{2}$ ) vacations.
In Table 1, we summarize all eight major optimality concepts according to a chosen dual classification: single versus multiple criteria and the extent of the 'given', ranging from 'all-but' to 'none-except'. Traditional concept of optimality, characterized by so many 'givens' and a single criterion, appears properly to be the farthest removed from real optimal conditions or circumstances for problem solving in the lower right corner of the table.

There are also other ways of classifying various optimality concepts as there are many mixed and intermediate situations of partially given and partially to be determined constraints and objectives. Only the upper left-hand corner represents a pure, non-mixed situation where all alternatives and a single criterion are all given $a$ priori. That happens to be the only situation many analysts, economists and mathematicians have chosen to pursue.

It is clear that the distance from the upper left-hand to the lower right-hand corner still remains as large as the gap between the reality and its model.

\section{References}

[1] M. Zeleny, Multiple Criteria Decision Making, McGrawHill, New York, 1982.

[2] M. Zeleny, Optimal system design with multiple criteria: de novo programming approach, Engineering Costs and Production Economics 10 (1986), 89-94.

[3] M. Zeleny, Trade-offs-free management via de novo programming, Int. J. Oper. Quant. Management 1 (1995), 3-13.

Milan ZELENY

Graduate School of Business

Fordham University at Lincoln Center

New York, NY 10023

USA 